\title{
Study of thermal aggregation and gelation of oat globulin by Raman spectroscopy
}

\author{
C.-Y. Ma ${ }^{\mathrm{a}, \mathrm{b}, *}$, M.K. Rout ${ }^{\mathrm{a}}$ and D.L. Phillips ${ }^{\mathrm{a}, \mathrm{b}, \mathrm{c}}$ \\ ${ }^{a}$ Food Science Laboratory, Department of Botany, The University of Hong Kong, Pokfulam Road, \\ Hong Kong, China \\ ${ }^{\mathrm{b}}$ Center for Applied Spectroscopy and Analytical Sciences, The University of Hong Kong, \\ Pokfulam Road, Hong Kong, China \\ ${ }^{\mathrm{c}}$ Department of Chemistry, The University of Hong Kong, Pokfulam Road, Hong Kong, China
}

\begin{abstract}
Thermal aggregation and gelation of oat globulin were studied by FT-NIR Raman spectroscopy. The buffer-soluble aggregates exhibited a Raman spectrum similar to that of the unheated control, whereas the insoluble aggregates showed intensity increases in the tryptophan, $\mathrm{C}-\mathrm{H}$ bending and $\mathrm{C}-\mathrm{H}$ stretching bands, and a decrease in the tyrosine doublet $\left(I_{850} / I_{830}\right)$, suggesting protein denaturation. However, analysis of the amide I region using Raman Spectral Analysis Package (RASP) program revealed marked decreases in $\alpha$-helical and increases in $\beta$-sheet structure in both soluble and insoluble aggregates. Similar conformational changes were also observed in the heat-induced oat globulin gels, and may be attributed to realignment of molecular segments and formation of intermolecular $\beta$-sheet structures. Thermal gelation under the influence of different chaotropic salts showed some shifts in band positions and changes in band intensity, following the lyotropic series of anions. Several protein structure perturbants, including sodium dodecyl sulfate, dithiothreitol, urea and sodium laurate, were found to affect the Raman spectral characteristics of oat globulin gels. The data suggest that changes in gelling properties of oat globulin by these chemicals may be related to conformational changes of the protein during gelation.
\end{abstract}

\section{Introduction}

Thermal aggregation and gelation are important functional properties of proteins affecting their uses in food systems [1]. Thermal aggregation (or coagulation) is the random interactions between protein molecules to form aggregates, while gelation is the formation of three-dimensional networks with some degree of order [2]. Most plant proteins are not heat-coagulable due to their relatively high heat stability [3]. Oat globulin, the major protein fraction in oats with quaternary structure similar to soy 11S globulin (glycinin), is heat-coagulable [4] and can form a self-supporting gel under appropriate conditions [5].

Since thermal aggregation and gelation are important protein properties, the study of conformational changes in proteins during these processes will provide crucial information for more effective utilization of these ingredients in various food systems. Since aggregation and gelation generally occur at high protein concentrations with the formation of opaque coagulum/gel, the structural changes cannot be studies by techniques such as NMR or circular dichroism (CD) spectroscopy. Vibrational spectroscopic techniques such as Raman and FT-IR have the advantage of being adaptable to a wide range of samples including liquids, powders, semi-solids and films [6]. However, fluorescence is a major problem with Raman spectroscopy and has limited its use in plant proteins. A significant advance in solving the fluorescence problem was the development of Fourier transform (FT) Raman system with the use of a diode-laser pumped Nd:YAG laser radiation [7,8].

\footnotetext{
*Corresponding author. Tel.: +852 2299 0318; Fax: +852 2858 3477; E-mail: macy@ hkucc.hku.hk.
} 
In a previous study, changes in the oat globulin conformation under the influence of different environmental conditions and heating were studied by FT-Raman spectroscopy [9]. In this investigation, changes in oat globulin conformation during thermal aggregation and gelation will be monitored by FT-Raman spectroscopy.

\section{Materials and methods}

Oat seeds (variety Hinoat) were grown in the Central Experimental Farm, Ottawa, Agriculture and Agri-Food Canada, and dehulled with a pin-mill. Oat globulin was extracted from defatted oat groats with $1 \mathrm{M} \mathrm{NaCl}$ [10], and the protein content, determined by a micro-Kjeldahl method [11] using a nitrogen to protein conversion factor of 5.80 , was $98.9 \%$. The purity of the oat globulin preparation was checked by SDS-polyacrylamide gel electrophoresis [12] and found to be highly homogeneous. All chemicals used were of analytical grade.

\subsection{Thermal aggregation}

To study thermal aggregation, $1 \%(\mathrm{w} / \mathrm{v})$ oat globulin dispersions were prepared in $0.01 \mathrm{M}$ phosphate buffer, $\mathrm{pH} 7.4$, containing $1.0 \mathrm{M} \mathrm{NaCl}$, and were heated in stoppered glass tubes at $110^{\circ} \mathrm{C}$ in an autoclave for $30 \mathrm{~min}$ [4]. The heat-aggregated protein was separated by centrifugation into buffer-soluble and insoluble fractions [4]. The two fractions were dialyzed against distilled water and freeze-dried.

\subsection{Thermal gelation}

To prepare heat-induced gels, oat globulin dispersions $(10 \% \mathrm{w} / \mathrm{v})$ were prepared in $0.2 \mathrm{M} \mathrm{NaCl}$, and $\mathrm{pH}$ was adjusted to 9.5 with $1 \mathrm{M} \mathrm{NaOH}$. Aliquots were heated in sealed test tubes at $100^{\circ} \mathrm{C}$ for $20 \mathrm{~min}$ [5]. Previous study [5] showed that oat globulin can only form heat-induced gels at alkaline $\mathrm{pH}$ where protein solubility is higher. Chaotropic salts were added to give a final salt concentration of 1.0 M. Protein structure perturbants including sodium dodecyl sulfate, dithiothreitol, urea and sodium laurate, were added as solid to the oat globulin dispersions to give the desirable final concentrations. The selected salt and perturbant concentrations were based on previous studies $[4,5,13,14]$ which showed that conformation of oat globulin was markedly affected under these conditions and that thermal gelation behavior was also altered.

\subsection{Raman spectroscopy}

Raman spectra were recorded with a Bio-Rad FTS-60 FT-NIR Raman spectrometer equipped with Nd:YAG laser at $1064 \mathrm{~nm}$ (Bio-Rad Lab., Cambridge, MA). Raman spectra were collected at room temperature under the following conditions: laser power, $500 \mathrm{~mW}$; spectral resolution, $4 \mathrm{~cm}^{-1}$; number of scans, 1000. The spectral data were baseline-corrected and normalized to the intensity of the phenylalanine band at $1004 \pm 1 \mathrm{~cm}^{-1}$. The Raman spectra were plotted as intensity (arbitrary units) against Raman shift in wavenumber $\left(\mathrm{cm}^{-1}\right)$. Protein dispersions containing urea were spiked with $0.2 \mathrm{M} \mathrm{KNO}_{3}$ since urea vibrates at the phenylalanine region, and the $\mathrm{KNO}_{3}$ peak $\left(1046 \pm 1 \mathrm{~cm}^{-1}\right)$ was used instead for normalization.

The secondary structure composition of the samples was estimated using the algorithm of Williams [15]. This was based on the averaged scans of the raw (not baseline corrected, smoothed or normalized) 
Raman spectra in the amide I region, using the RASP (Raman Spectral Analysis Package) program (version 2.1) of Przybycien and Bailey [16].

All analyses were performed in duplicates or triplicates, and the results are reported as the average of these replicates.

\section{Results and discussion}

Raman spectra of heated oat globulin dispersions or wet pellets were found to be noisy with very poor signal to noise ratio (see Fig. 3d). The heated protein samples were therefore freeze-dried, and Raman spectra of solid samples were recorded. Previous study [9] showed that freeze-dried protein samples exhibited Raman spectra identical to those in dispersions or wet pellets indicating that freeze drying did not affect the conformation of oat globulin. Freeze-dried oat globulin was therefore used as a control instead of $10 \%$ dispersions in distilled water used previously [9].

\subsection{Spectral assignment}

Figure 1a shows a typical Raman spectrum of freeze-dried oat globulin. Table 1 shows the tentative assignment of some major bands based on comparison with Raman spectral data reported by other researchers [17-19]. The locations of the amide I and III peaks and RASP analysis (Table 2) show that $\beta$-types (sheets and turns) and random coils are the major secondary structures in oat globulin. This is in agreement with $\mathrm{CD}$ data which indicate that oat globulin, similar to most plant globulins, has a relatively

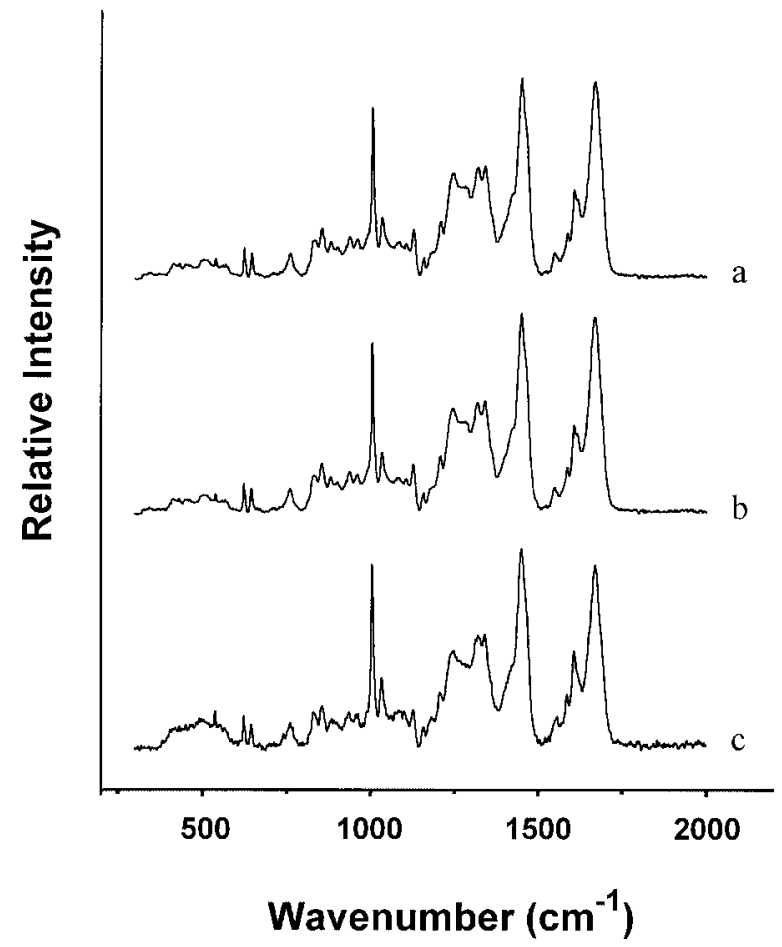

Fig. 1. Raman spectra (450-2000 $\mathrm{cm}^{-1}$ ) of (a) oat globulin powder, (b) freeze-dried buffer-soluble aggregates, and (c) freeze-dried buffer-insoluble aggregates. 
Table 1

Tentative assignment of some bands in the Raman spectrum of oat globulin (in 0.01 M phosphate buffer, $\mathrm{pH}$ 7.4)

\begin{tabular}{lll}
\hline Wave-number, $\mathrm{cm}^{-1}$ & Assignment & Structural information \\
\hline 760 & tryptophan & sharp intense line for buried structure \\
830,850 & tyrosine & state of phenol-OH (exposed or buried, H donor or receptor) \\
$>1275$ & amide III' & $\alpha$-helix \\
$1235 \pm 5$ & amide III' & antiparallel $\beta$-sheet \\
$1245 \pm 4$ & amide III' $^{\prime}$ & disordered structure \\
1250 & C-H stretching $_{1255 \pm 5}$ & microenvironment, polarity \\
$1270 \pm 3$ & amide I' & $\alpha$-helix \\
$1265 \pm 3$ & amide I' & antiparallel $\beta$-sheet \\
$2800-3000$ & amide I' & disordered structure \\
\hline
\end{tabular}

Table 2

Secondary structure composition of oat globulin gel, soluble aggregates and insoluble aggregates ${ }^{1}$

\begin{tabular}{lccc}
\hline Sample & $\alpha$-helix (\%) & $\beta$-sheet (\%) & Random coil $/ \beta$-turn (\%) \\
\hline Control (no pH adjustment, unheated) & 24 & 16 & 60 \\
Control (pH 9.5, unheated) & 18 & 29 & 53 \\
Gel (pH 9.5, heated) & 14 & 58 & 28 \\
Soluble aggregates & 14 & 62 & 24 \\
Insoluble aggregates & 17 & 61 & 22 \\
\hline
\end{tabular}

${ }^{1}$ Determined by Raman spectral analysis of the amide I region. Averages of duplicate determinations.

small quantity of $\alpha$-helical structure but a large amount of $\beta$-type and random coil structures [20]. The small intensity of the transitions near $500 \mathrm{~cm}^{-1}$ makes it difficult to analyze the disulfide peaks. This is attributed to the relatively low disulfide and sulfhydryl contents in oat globulin, similar to that of other legume globulins [21-23].

\subsection{Raman spectra of heat-induced aggregates}

The Raman spectra of heat-induced buffer-soluble and insoluble aggregates were shown in Fig. 1b and $1 \mathrm{c}$, respectively. The results show that heat aggregation did not lead to marked shifts in the positions of the major Raman bands. However, changes in the normalized intensity of several Raman bands were observed in the buffer-insoluble aggregates (Fig. 2). There were marked increases in the tryptophan, amide I and III, and the $\mathrm{CH}$ bending and stretching bands, and a slight decrease in the intensity ratio of the doublet tyrosine band at $850 \mathrm{~cm}^{-1}$ and $830 \mathrm{~cm}^{-1}, I_{850} / I_{830}$. However, Raman spectral analysis of the amide I region shows that both soluble and insoluble aggregates have much lower $\alpha$-helix and higher $\beta$-sheet contents than the unheated control (Table 2).

Exposure of buried tryptophan residues in proteins has been observed by the decrease in peak intensity around $760 \mathrm{~cm}^{-1}$ [24]. An increase in tryptophan peak intensity suggests burying of the residues in the aggregated protein. The intensity ratio of the $I_{850} / I_{830}$ band can be used to monitor the microenvironment of the tyrosine side chain [17]. A decrease in the tyrosine doublet band intensity suggests an increased "buriedness" or participation of the tyrosine phenolic groups as hydrogen bond donors [25]. The increases in intensity in the amide I, amide III and $\mathrm{C}-\mathrm{H}$ stretching and bending transitions in the insoluble aggregates indicate protein denaturation [9]. 


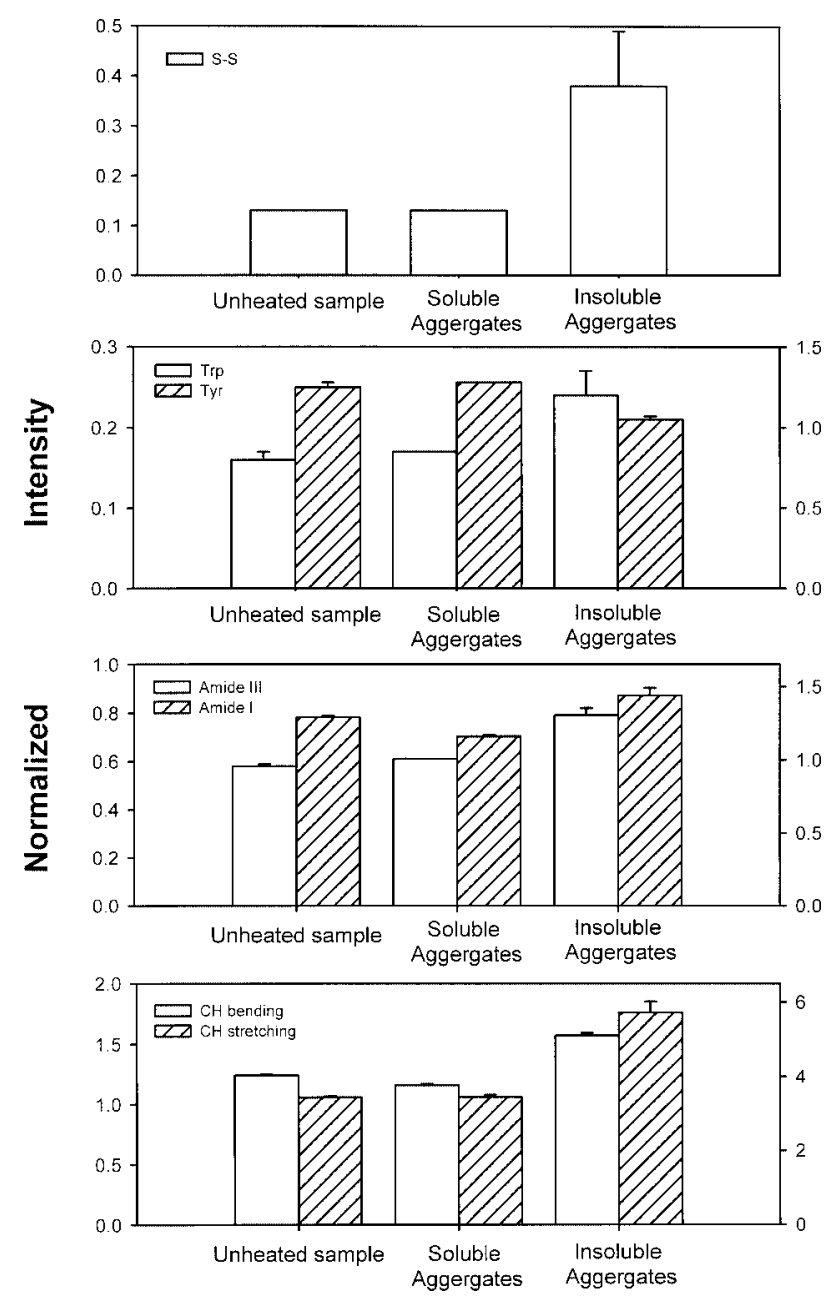

Fig. 2. Normalized intensity of several regions in the Raman spectrum of oat globulin powder (unheated control), buffer-soluble aggregates and buffer-insoluble aggregates. Error bars represent standard deviations of the means.

The data suggest that the soluble aggregates contained relatively native protein while protein in the insoluble aggregate fraction was extensively denatured. Differential scanning calorimetric (DSC) study [14] and FT-IR spectroscopy [26] also showed a redistribution of native and denatured protein in the soluble and insoluble oat globulin aggregates, respectively.

\subsection{Thermal gelation}

At pH 9.5 (Fig. 3b), oat globulin showed a Raman spectrum different from that at neutral pH (Fig. 3a), with significant shift in some bands, particularly the amide III and the $\mathrm{C}-\mathrm{H}$ bending transitions. Shift in Raman bands at both alkaline and acidic pHs was also observed in our previous study [9] and was attributed to partial protein denaturation. Heat-induced oat globulin gel samples (Fig. 3c and 3d) did not show further shift in Raman bands. The wet gel sample (Fig. 3d) showed a noisy pattern but the spectrum is almost identical to that of the freeze-dried sample (Fig. 3c). Figure 4 shows the effect of gelation on the normalized intensity of several Raman bands. When compared to the unheated control (at $\mathrm{pH} 7.4)$, 


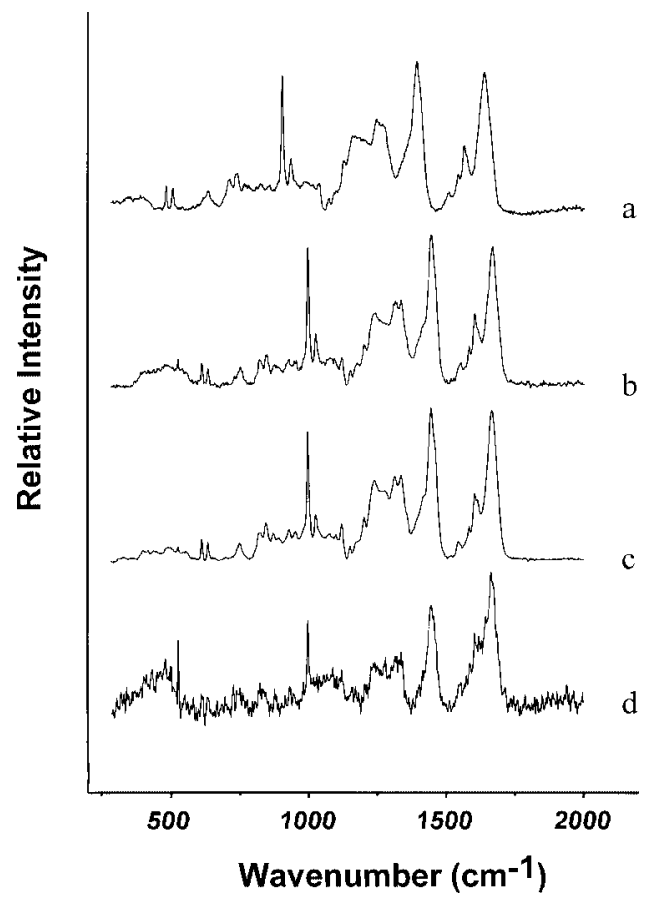

Fig. 3. Raman spectra of (a) oat globulin powder (no $\mathrm{pH}$ adjustment), (b) freeze-dried oat globulin dispersion (10\%) with $\mathrm{pH}$ adjusted to 9.5 , (c) freeze-dried oat globulin gel (prepared by heating $10 \%$ oat globulin dispersion, $\mathrm{pH} 9.5$, at $100^{\circ} \mathrm{C}$ for $20 \mathrm{~min}$ ), and (d) wet packed oat globulin gel.

there were slight decreases in the tryptophan, tyrosine doublet, amide I and $\mathrm{C}-\mathrm{H}$ bending transitions, and an increase in $\mathrm{C}-\mathrm{H}$ stretching band. However, these changes were likely attributed to a change in $\mathrm{pH}$ since the unheated control (at $\mathrm{pH}$ 9.5) showed peak intensities (data not shown) similar to that of the gel sample. Raman spectral analysis of the amide I region shows that increase in $\mathrm{pH}$ led to a decrease in $\alpha$ helical and increase in $\beta$-sheet structures, suggesting protein unfolding (Table 2). Gel samples exhibited further decrease in $\alpha$-helix and a marked increase in $\beta$-sheet content.

Decreases in the intensity of the aromatic amino acid residue transitions were also observed in heatinduced whey protein gels [27] and were attributed to exposure of these residues which may play a role in hydrophobic interactions. Changes in intensity of the amide and $\mathrm{C}-\mathrm{H}$ bands suggest changes in protein conformation [9]. The results show that heat-induced gelation led to further changes in the secondary structure of oat globulin. The data are consistent with our previous DSC study which show that when oat globulin dispersions were heated under conditions that induce gelation, there was a slight decrease in denaturation enthalpy, indicating further protein unfolding in addition to that caused by $\mathrm{pH}$ adjustment, but the heat-induced gel sample still exhibited native structure as shown by an enthalpy value more than half of the unheated control [5]. It has been suggested that thermal gelation of protein involves conformational rearrangement and realignment of molecular segments, including perhaps the formation of intermolecular $\beta$-sheet structures [28]. The marked increases in $\beta$-sheet contents in both the thermal aggregates and gels (Table 2 ) may be attributed to these molecular changes.

The effects of a number of chemical reagents known to cause changes in the conformation of oat globulin and influence its gelation properties on Raman characteristics were studied. Figures 5 and 6 show the Raman spectra and normalized intensity of selected Raman bands, respectively, of oat globulin gels 


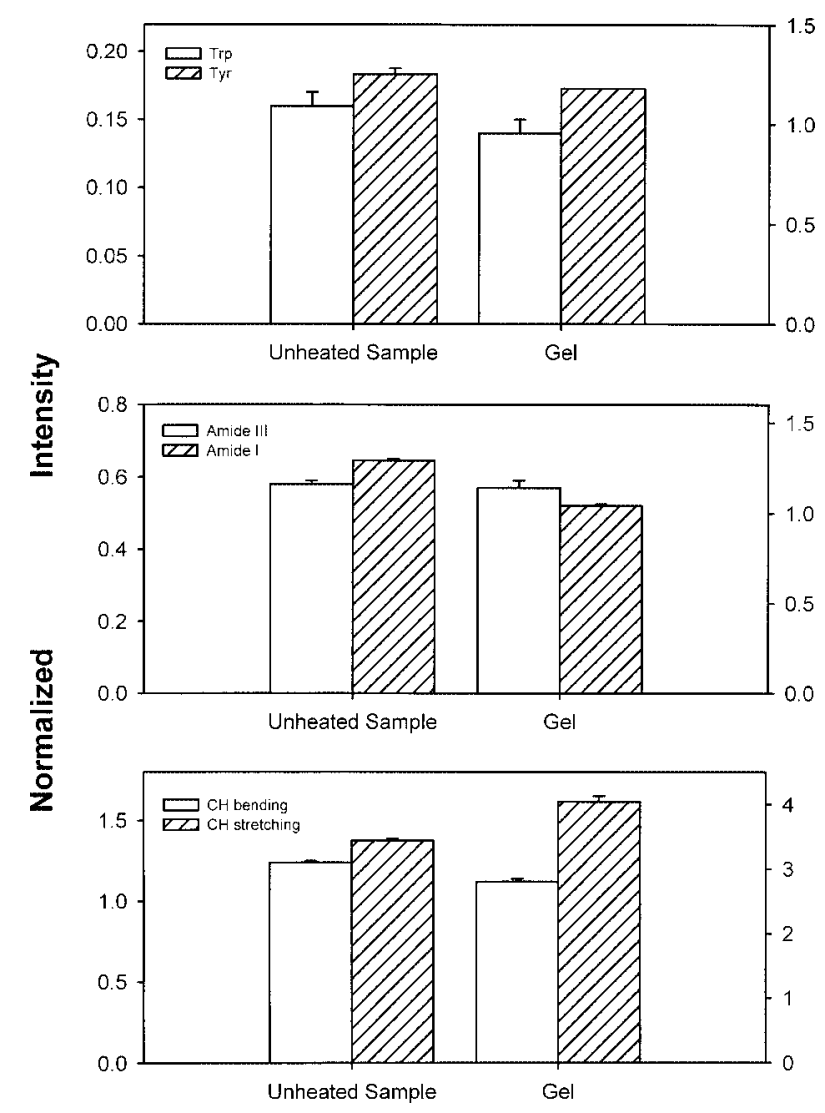

Fig. 4. Normalized intensity of several regions in the Raman spectrum of oat globulin powder (unheated control, $\mathrm{pH}$ unadjusted) and freeze-dried oat globulin gel. Error bars represent standard deviations of the means.

formed in the presence of several chaotropic salts. Slight shifts in the amide I and amide III transitions to higher wavenumbers were observed when the anion was changed from chloride (Fig. 5a) to bromide (Fig. 5b), iodide (Fig. 5c) and thiocyanide (Fig. 5d). Progressive changes in Raman band intensities were observed (Fig. 6), following the lyotropic anion series [29].

Salts can perturb protein conformation by affecting electrostatic and hydrophobic interactions [30,31]. The level of influence depends on the nature of anions and cations, following the lyotropic series [29]. Anions higher in the series (e.g., $\mathrm{I}^{-}$and $\mathrm{SCN}^{-}$) could weaken intramolecular hydrophobic interactions and enhance the unfolding tendency of proteins [32]. Previous study [9] also showed similar changes in Raman characteristics of oat globulin by these salts, indicating progressive protein unfolding. Addition of these chaotropic salts also led to progressive increases in gel hardness which suggests that more extensive aggregation and gelation can be promoted by perturbing the conformation of oat globulin [5].

The effect of several protein structure perturbants on the Raman spectral characteristics of oat globulin gels is shown in Figs 7 and 8. Shifts in both amide I and amide III bands were observed with the addition of $10 \mathrm{mM}$ sodium dodecyl sulfate (SDS) (Fig. 7b), $10 \mathrm{mM}$ dithiothreitol (DTT) (Fig. 7c), $6 \mathrm{M}$ urea (Fig. 7d) and $1 \%$ fatty acid salt, sodium laurate (Fig. 7e). These perturbants also led to increases in the intensity of the major Raman transitions (Fig. 8). The results indicate that these chemicals cause marked changes in the structure and conformation of oat globulin during heat-induced gel formation. 


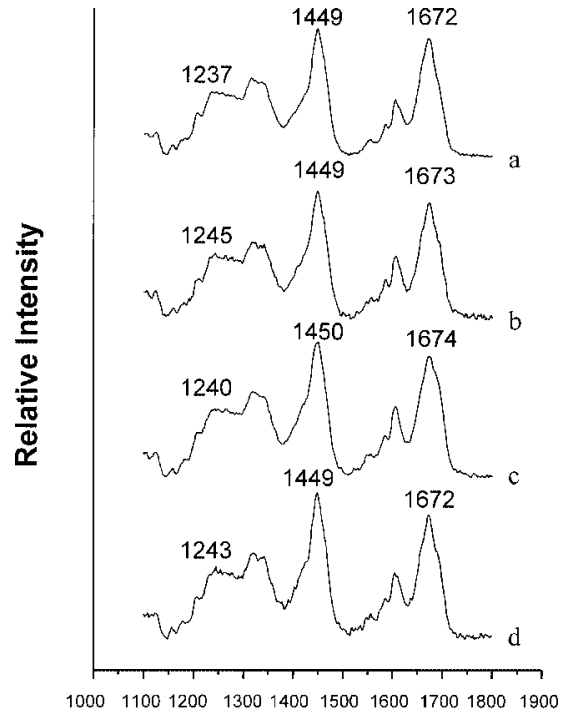

Wavenumber $\left(\mathrm{cm}^{-1}\right)$

Fig. 5. Raman spectra $\left(1100-1800 \mathrm{~cm}^{-1}\right)$ of freeze-dried oat globulin gels prepared from $10 \%$ dispersions with $1 \mathrm{M}$ salts. (a) $\mathrm{NaCl}$, (b) $\mathrm{NaBr}$, (c) $\mathrm{NaI}$, and (d) $\mathrm{NaSCN}$.

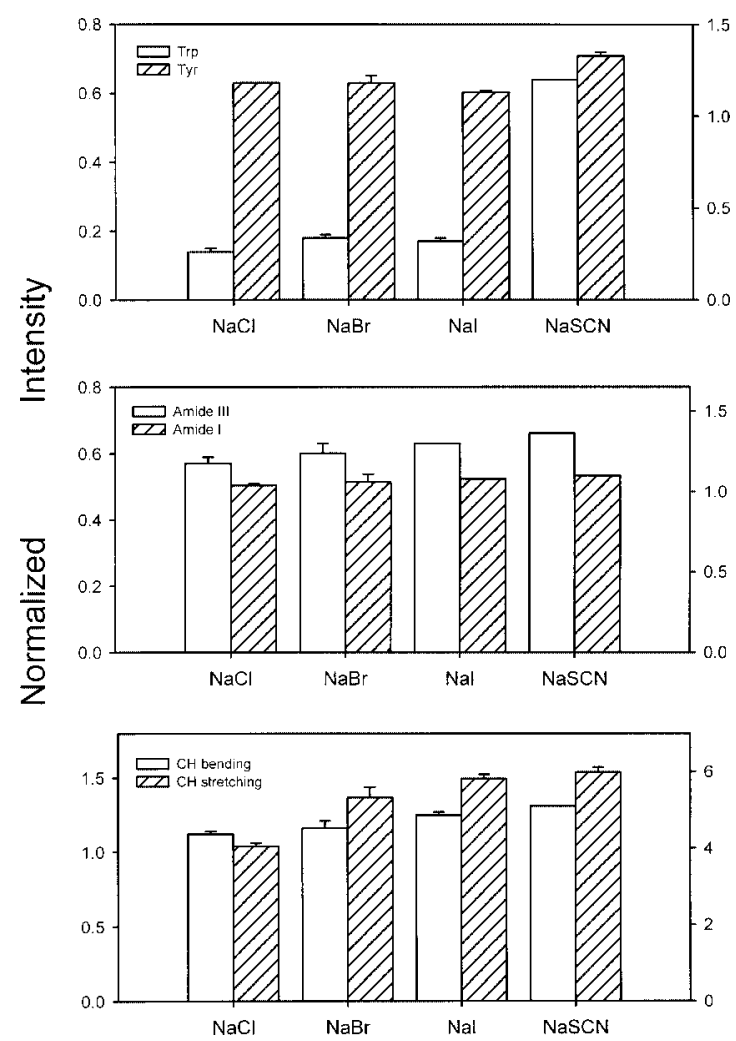

Fig. 6. Effect of chaotropic salts $(1.0 \mathrm{M})$ on normalized intensity of several regions in the Raman spectrum of freeze-dried oat globulin gels. Error bars represent standard deviations of the means. 


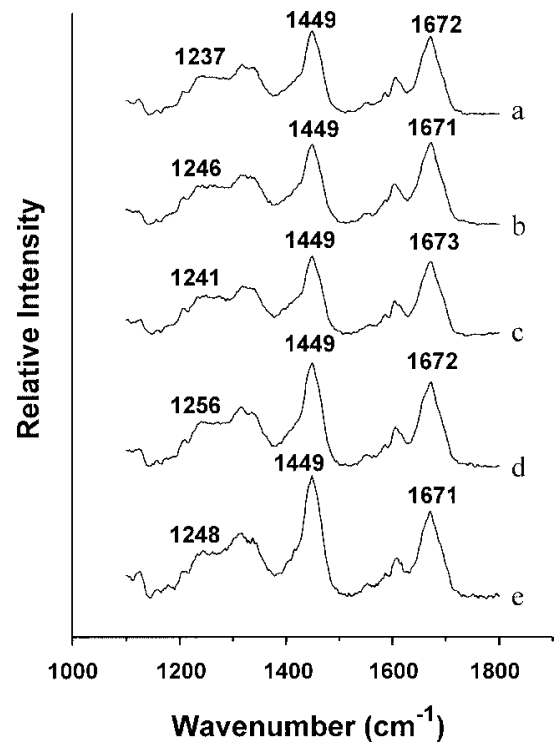

Fig. 7. Effect of different protein perturbants on the Raman spectrum of freeze-dried oat globulin gel. (a) Control gel (no additive), (b) $10 \mathrm{mM}$ sodium dodecyl sulfate, (c) $10 \mathrm{mM}$ dithiothreitol, (d) $6 \mathrm{M}$ urea, and (e) $1 \%$ sodium laurate.

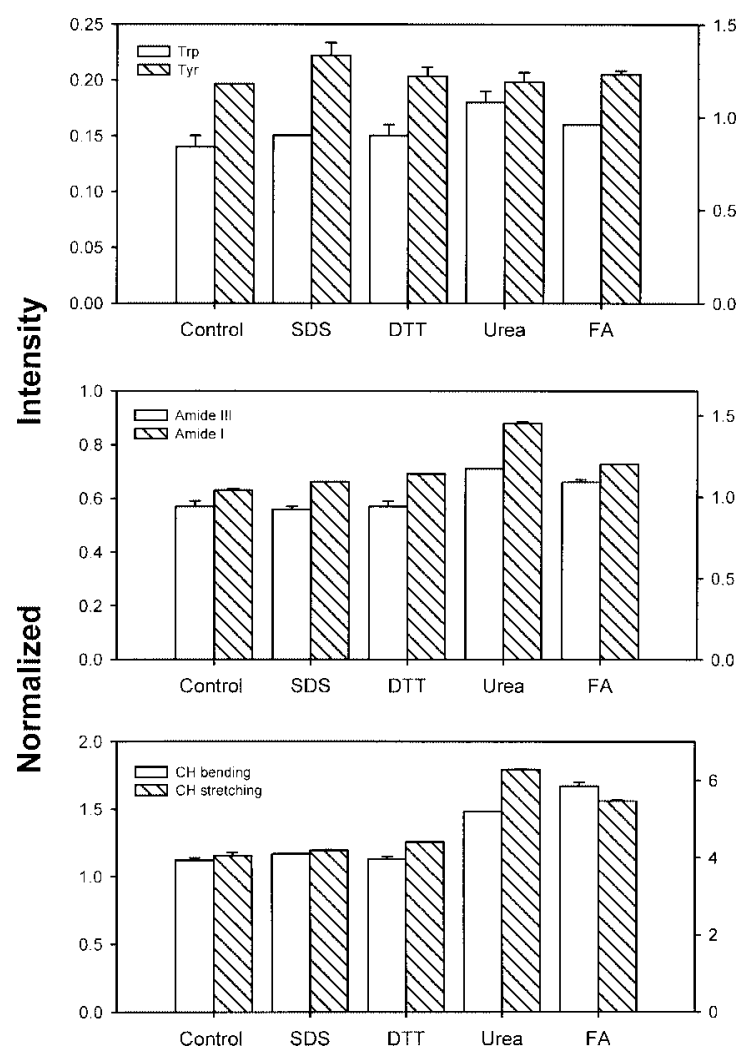

Fig. 8. Effect of different protein perturbants on the normalized intensity of several regions in Raman spectrum of freeze-dried oat globulin gel. Perturbants: no additive (control gel), $10 \mathrm{mM}$ sodium dodecyl sulfate (SDS), $10 \mathrm{mM}$ dithiothreitol (DTT), $6 \mathrm{M}$ urea (urea) and $1 \%$ sodium laurate (FA). Error bars represent standard deviations of the means. 
SDS is an anionic detergent which binds to protein by non-covalent forces to increase the net charge leading to ionic repulsion and protein unfolding [33]. DTT, a reducing agent, can breakup disulfide bonds and dissociate oligomeric proteins into their subunits. Urea effectively disrupts the hydrogen-bonded structure of water and facilitates protein unfolding by weakening hydrophobic interactions [34]. Urea also increases the "permitivity" of water [35] for the apolar residues causing loss of protein structure and heat stability. Previous Raman study [9] showed that these reagents caused marked changes in oat globulin conformation. This could be due to perturbation of the tertiary and quaternary structures of the oligomeric protein by destabilizing some primary (hydrogen bonds, hydrophobic forces) and secondary (disulfide bonds) chemical forces which are important in the stabilization of oat globulin conformation. These chemical forces also play an important role in the thermal gelation process, and previous report [5] shows that SDS, DTT and urea all led to marked decreases in gel hardness. Fatty acid salts such as sodium laurate have been found to improve gel-forming ability and reduce thermal stability of myosin [36] and oat globulin [5] due to binding of these amphiphiles to protein, causing repulsion between protein chains and a more ordered gelation upon heat treatment.

\section{Conclusions}

The present study shows that heat treatments used to induce aggregation and gelation did not cause marked changes in the Raman spectral characteristics of oat globulin, probably due to the relatively high thermal stability of the protein [13]. However, the addition of chemicals known to perturb protein conformation led to marked changes in Raman characteristics of oat globulin gels. The present data, together with previous studies, indicate that by modifying the protein conformation, these perturbants can either enhance or inhibit the thermal gelation of oat globulin. This study also shows that FT-Raman spectroscopy is an appropriate technique to study aggregation and gelation of plant proteins such as oat globulin.

\section{Acknowledgements}

The research project was supported by a Hong Kong Research Grants Council (RGC) grant (HKU 7089/99M), a Hong Kong University Research and Conference grant, and a Hong Kong University Science Faculty Collaborative Seed fund. We would like to thank Dr. V.D. Burrows, Eastern Cereal and Oilseed Research Centre, Agriculture and Agri-Food Canada, for the supply of dehulled oat seeds.

\section{References}

[1] J.E. Kinsella, Functional properties of food proteins: A review, CRC Critical Reviews of Food Science and Nutrition 7 (1976), 219-280.

[2] A.-M. Hermansson, in: Functionality and Protein Structure, A. Pour-EL, ed., ACS Symposium Series 92, American Chemical Society, Washington, DC, 1979, pp. 81-104.

[3] B. German, S. Damordaran and J.E. Kinsella, Thermal dissociation and association behavior of soy proteins, Journal of Agricultural and Food Chemistry 30 (1982), 807-812.

[4] C.-Y. Ma and V.R. Harwalkar, Thermal coagulation of oat globulin, Cereal Chemistry 4 (1987), 212-218.

[5] C.-Y. Ma, G. Khanzada and V.R. Harwalkar, Thermal gelation of oat globulin, Journal of Agricultural and Food Chemistry 36 (1988), 275-280.

[6] E.C.Y. Li-Chan, Methods to monitor process-induced changes in food proteins: an overview, in: Process-Induced Chemical Changes in Foods, F. Shahidi, C.T. Ho and N. Chuyen, eds, Plenum Press, New York, 1998, pp. 5-23. 
[7] B. Schrader, A. Hoffman, A. Simon and J. Sawatzki, Can a Raman renaissance be expected via the near-infrared Fourier transform technique? Vibrational Spectroscopy 1 (1991), 239-250.

[8] E.C.Y. Li-Chan, S. Nakai and M. Hirotsuka, Raman spectroscopy as a probe of protein structure in food systems, in: Protein Structure-Function Relationships in Foods, R.Y. Yada, R.L. Jackson and L.L. Smith, eds, Blackie Academic, London, 1994, pp. 163-197.

[9] C.-Y. Ma, M.K. Rout, W.M. Chan and D.L. Phillips, Raman spectroscopy study of oat globulin conformation, Journal of Agricultural and Food Chemistry 48 (2000), 1542-1547.

[10] C.-Y. Ma and V.R. Harwalkar, Chemical characterization and functionality assessment of oat protein fractions, Journal of Agricultural and Food Chemistry 32 (1984), 144-149.

[11] J.M. Concon and D. Soltess, Rapid micro-Kjeldahl digestion of cereal grains and other biological materials, Analytical Biochemistry 53 (1973), 35-41.

[12] U.K. Laemmli, Cleavage of structural proteins during the assembly of the head of bacteriophage $\mathrm{T}_{4}$, Nature 227 (1970), $680-685$.

[13] V.R. Harwalkar and C.-Y. Ma, Study of thermal properties of oat globulin by differential scanning calorimetry, Journal of Food Science 52 (1987), 394-398.

[14] C.-Y. Ma and V.R. Harwalkar, Study of thermal denaturation of oat globulin by differential scanning calorimetry, Journal of Food Science $\mathbf{5 3}$ (1988), 531-534.

[15] R.W. Williams, Estimation of protein secondary structure from the laser amide I spectrum, Journal of Molecular Biology 1521 (1983), 783-813.

[16] T.M. Przybycien and J.E. Bailey, Structure-function relationships in the Inorganic salt-induced precipitation of $\alpha$ chymotrypsin, Biochimica et Biophysica Acta 995 (1989), 231-245.

[17] A.T. Tu, Peptide backbone conformation and microenvironment of protein side-chains, in: Spectroscopy of Biological Systems, R.J.H. Clark and R.E. Hester, eds, John Wiley and Sons, New York, NY, 1986, pp. 47-112.

[18] W.L. Peticolas, Raman spectroscopy of DNA and proteins, Methods in Enzymology 226 (1995), 389-416.

[19] E.C.Y. Li-Chan and L. Qin, The application of Raman spectroscopy to the structural analysis of food protein networks, in: Paradigm for Successful Utilization of Renewable Resources, D.J. Sessa and J.L. Willett, eds, AOCS Press, Champaign, Illinois, 1998, pp. 123-139.

[20] M.F. Marcone, Y. Kakuda and R.Y. Yada, Salt-soluble seed globulins of dicotyledonous and monocotyledonous plants II. Structural characterization, Food Chemistry 63 (1998), 265-274.

[21] E. Derbyshire, D.J. Wright and D. Boulter, Legumin and vicilin, storage proteins of legume seeds, Phytochemistry 15 (1976), 3-24.

[22] A.C. Brinegar and D.M. Peterson, Separation and characterization of oat globulin polypeptides, Archives in Biochemistry and Biophysics 219 (1982), 71-79.

[23] N.C. Neilsen, The structure and complexity of $11 \mathrm{~S}$ polypeptides in soybeans, Journal of American Oil Chemists' Society 62 (1985), 1680-1686.

[24] T. Kitagawa, T. Azuma and K. Hamaguchi, The Raman spectra of Bence-Jones proteins. Disulfide stretching frequencies and dependence of Raman intensity of tryptophan residues on their environments, Biopolymers 18 (1979), 451-465.

[25] E.C.Y. Li-Chan, Macromolecular interactions of food proteins studied by Raman spectroscopy: Interactions of $\beta$ lactoglobulin, $\alpha$-lactoglobulin, and lysozyme in solution, gels, and precipitates, in: Macromolecular Interactions in Food Technology, N. Parris, A. Kato, L.K. Creamer and J. Pearce, eds, American Chemical Society, Washington, DC, 1996, pp. $15-36$.

[26] C.-Y. Ma, M.K. Rout and W.-Y. Mock, Study of oat globulin conformation by Fourier transform infrared spectroscopy, Journal of Agricultural and Food Chemistry 49 (2001), 3328-3334.

[27] M. Nonaka, E. Li-Chan and S. Nakai, Raman spectroscopic study of thermally induced gelation of whey proteins, Journal of Agricultural and Food Chemistry 41 (1993), 1176-1181.

[28] P.C. Painter and J.L. Koenig, Raman spectroscopic study of the proteins of egg white, Biopolymers 15 (1976), 2155-2166.

[29] Y. Hatefi and W.G. Hanstein, Solubilization of particulate proteins and nonelectrolytes by chaotropic agents, Proceedings of National Academy of Science 62 (1969), 1129-1136.

[30] P.H. von Hippel and T. Scheich, The effect of neutral salts on the structure and conformational stability of macromolecules in solution, in: Structure and Stability of Biological Macromolecule, Vol. 2, S.N. Timesheff and G.D. Fasman, Marcel Dekker, New York, 1969, pp. 417-574.

[31] S. Damodaran and J.E. Kinsella, Effects of ions on protein conformation and functionality, in: Food Protein Deterioration, Mechanisms and Functionality, J.P. Cherry, ed., ACS Symposium Series 206, American Chemical Society, Washington, DC, 1982, pp. 327-357.

[32] P.H. von Hippel and K.Y. Wong, Neutral salts: The generality of their effects on the stability of macromolecular conformations, Science 145 (1964), 577-580.

[33] J. Steinhardt, The nature of specific and non-specific interactions of detergent with protein: complexing and unfolding, in: Protein-Ligand Interactions, H. Sund and G. Blauer, eds, W. de Gruyter, Berlin, Germany, 1975, pp. 412-426. 
[34] J.E. Kinsella, Relationship between structure and functional properties of food proteins, in: Food Proteins, P.F. Fox and J.J. Cowden, eds, Applied Science Publisher, London, 1982, pp. 51-103.

[35] F. Franks and D. England, The role of solvent interactions in protein conformation, CRC Critical Reviews in Biochemistry 3 (1975), 165-219.

[36] B. Egelandsdal, K. Fretheim and O. Harbitz, Fatty acid salts and analogs reduce thermal stability and improve gel formability of myosin, Journal of Food Science $\mathbf{5 0}$ (1965), 1399-1404. 


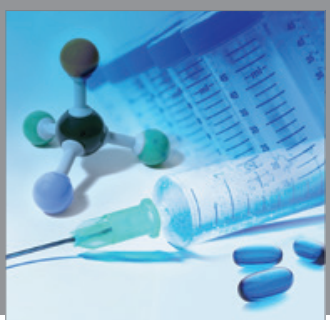

International Journal of

Medicinal Chemistry

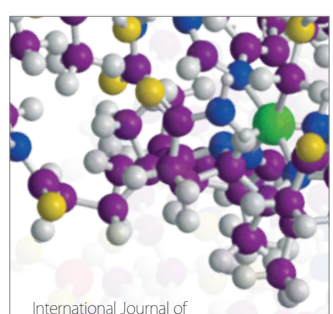

Carbohydrate Chemistry

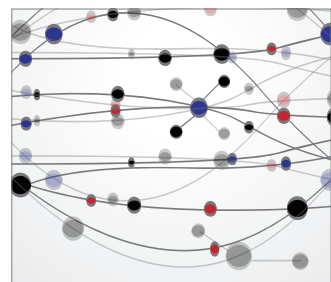

The Scientific World Journal
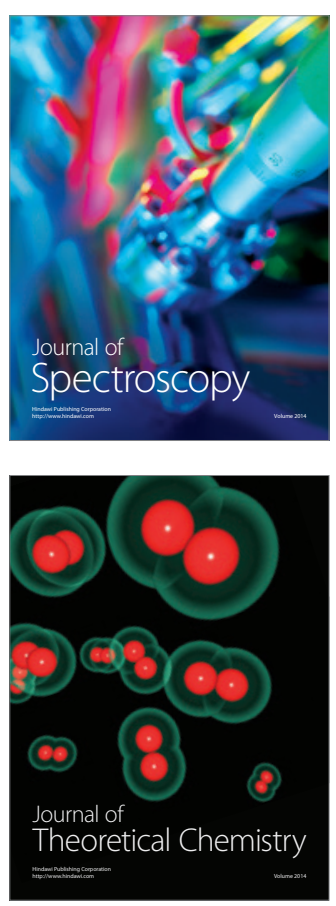
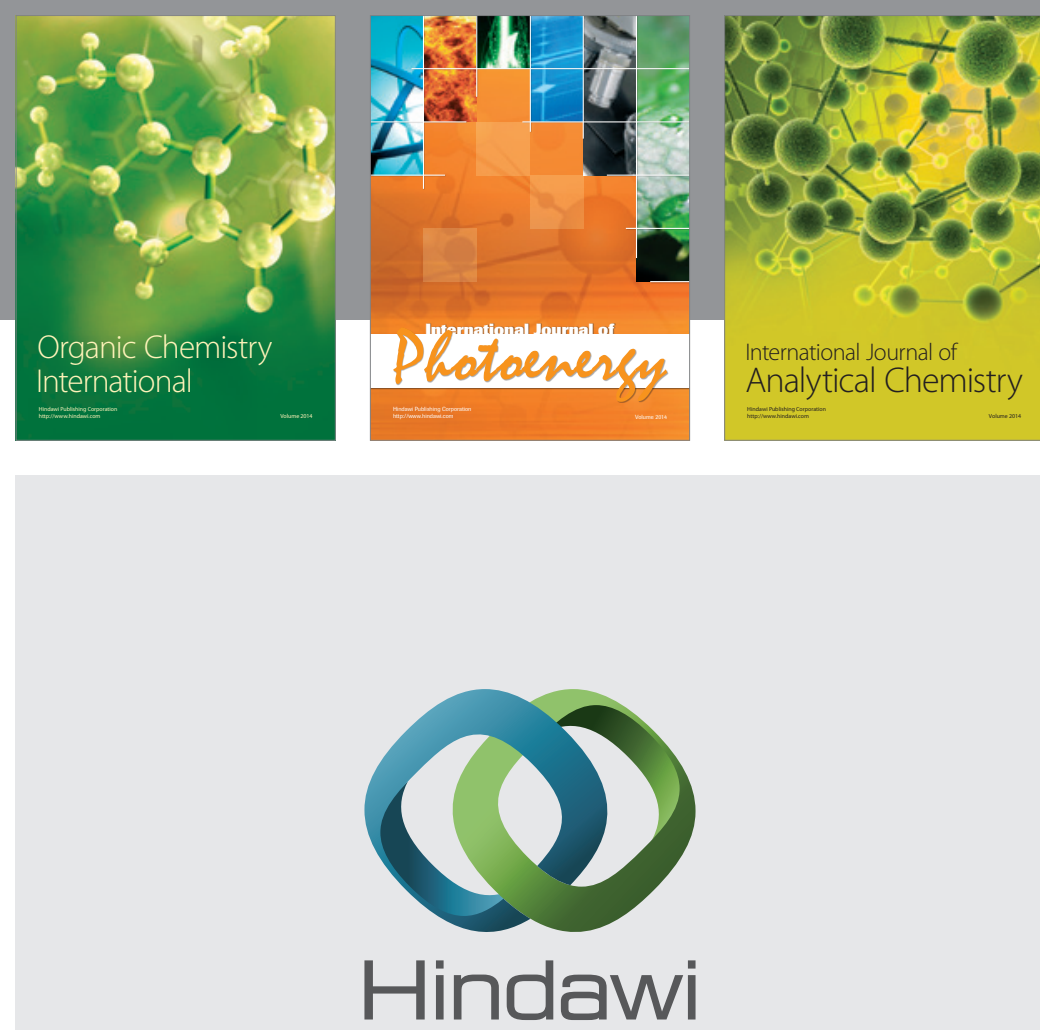

Submit your manuscripts at

http://www.hindawi.com
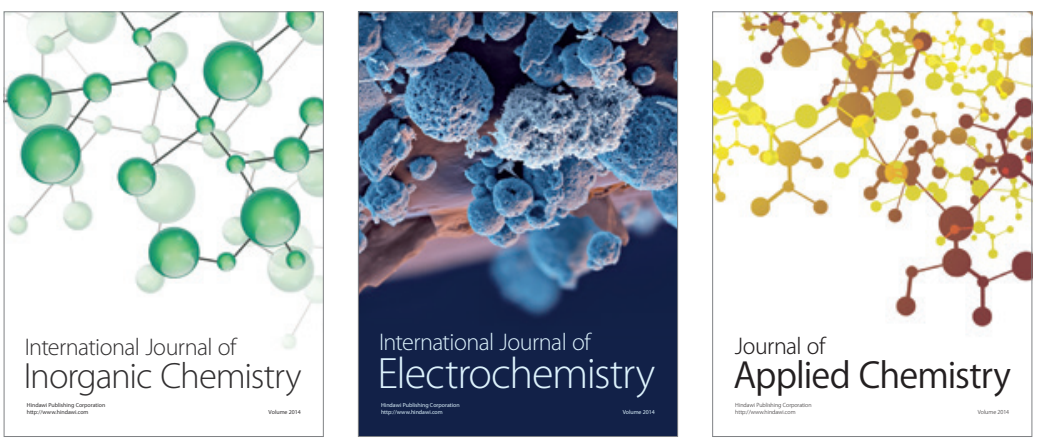

Journal of

Applied Chemistry
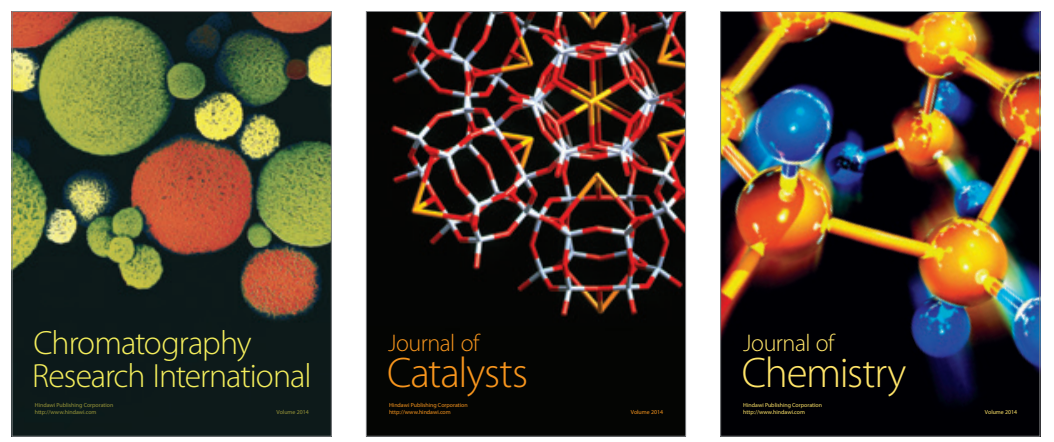
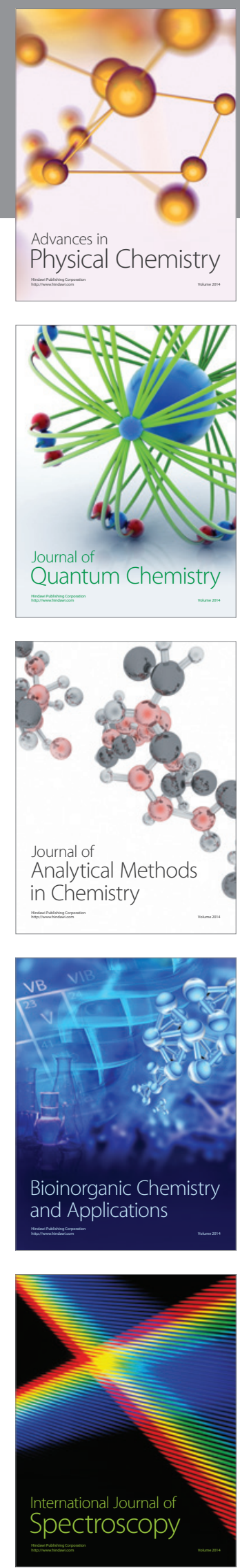